\title{
Video Article \\ Multiplex Cytokine Profiling of Stimulated Mouse Splenocytes Using a Cytometric Bead-based Immunoassay Platform
}

\author{
Jason S. Lehmann ${ }^{1}$, Amy Zhao ${ }^{1}$, Binggang Sun ${ }^{1}$, Weiping Jiang ${ }^{1}$, Shaoquan $\mathrm{Ji}^{1}$ \\ ${ }^{1}$ BioLegend
}

Correspondence to: Jason S. Lehmann at jlehmann@biolegend.com

URL: https://www.jove.com/video/56440

DOI: doi: $10.3791 / 56440$

Keywords: Immunology, Issue 129, Bead-based Immunoassay, Multiplex, Soluble Analyte, Flow Cytometry, Cytokines, Cytokinome

Date Published: 11/9/2017

Citation: Lehmann, J.S., Zhao, A., Sun, B., Jiang, W., Ji, S. Multiplex Cytokine Profiling of Stimulated Mouse Splenocytes Using a Cytometric Beadbased Immunoassay Platform. J. Vis. Exp. (129), e56440, doi:10.3791/56440 (2017).

\section{Abstract}

Bead-based immunoassays employ the same basic principle as sandwich immunoassays. Capture beads, which can be differentiated by size and internal allophycocyanin (APC) fluorescence intensity, are conjugated to antibodies specific to a particular analyte. Next, a selected panel of defined capture bead sets is incubated with a biological sample containing target analytes specific to the capture antibodies. A biotinylated detection antibody cocktail is added, which leads to the formation of capture bead-analyte-detection antibody sandwiches.

Finally, streptavidin-phycoerythrin (SA-PE) is added, which binds to biotinylated detection antibodies, providing fluorescent signal intensities in proportion to the amount of bound analyte. The PE fluorescent signal of analyte-specific beads regions is quantified using flow cytometry, and the concentrations of particular analytes are determined using data analysis software and the standard curve generated in the assay.

In this experiment, we use a mouse T helper cytokine panel to simultaneously quantify the concentration of 13 separate cytokine targets in tissue culture supernatants collected from mouse splenocytes cultured under various stimulatory conditions.

\section{Video Link}

The video component of this article can be found at https://www.jove.com/video/56440/

\section{Introduction}

In their role as soluble signaling molecules, cytokines mediate the highly coordinated and multifactorial processes that govern host immunological responses. They are expressed during all stages of the inflammatory process, from initiation to resolution, and regulate a complex interaction network that includes their own synthesis and that of their cellular receptors ${ }^{1}$. This communication network is layered with a complexity that goes beyond the synergistic or antagonistic relationships that may exist between individual components. Indeed, many cytokines are known to share redundant or at least partially overlapping functions ${ }^{2,3}$.

Integrated systems biology approaches that simultaneously quantify multiple cytokine analytes are currently providing an increasingly comprehensive understanding of the unique cytokinomes that orchestrate the immune responses underlying multiple disease states ${ }^{4,5}$. These disease states range from generalized inflammation to cancer, neuro-degenerative conditions, and cardiovascular disease ${ }^{6,7,8,9}$.

Such cytokine networks can be interrogated effectively using LEGENDplex bead-based immunoassays. These assays are based on the same principle as the sandwich Enzyme-Linked Immunosorbent Assay (ELISA), and use fluorescence encoded microspheres with capture antibodies covalently attached to their surface. These antibodies are immobilized on surface areas much smaller than those required by traditional ELISA formats. This enables such assays to be performed with far less sample volume, while at the same time reducing non-specific binding and providing multiplexed analysis of several analytes.

The assay described in this protocol utilizes this technology to quantitate up to 13 targets simultaneously. Data from this assay can be obtained using a wide variety of commonly available flow cytometers and unlike other available assays does not require the use of dedicated assayspecific instrumentation. With an expanding catalog of validated analyte panels, these assays have been used in several ongoing biomedical research projects ${ }^{10,11,12,13}$

To demonstrate the ease and utility of the assay format, in this experiment we use a Mouse T helper cytokine panel to quantitate 13 separate cytokine concentrations from tissue culture supernatants obtained from mouse splenocytes cultured under multiple stimulatory conditions. In addition to tissue culture supernatants, this assay can also be performed using serum or plasma samples. 


\section{Protocol}

All animal experiments were performed according to NIH recommendations contained within the Guide for the Care and Use of Laboratory Animals and were approved by the IACUC at BioLegend.

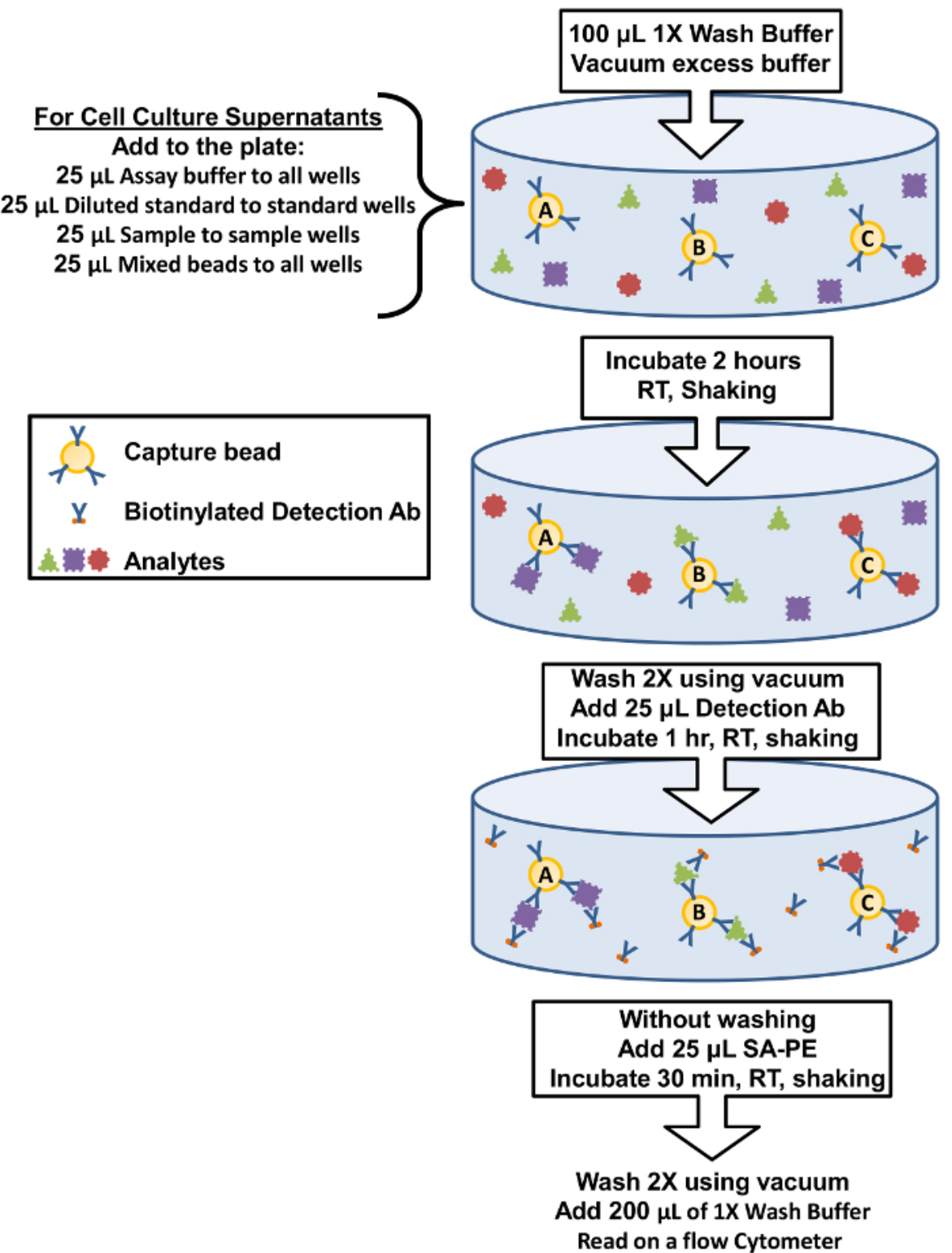

Figure 1: Filter Plate Assay Procedure Summary. The protocol for performing the assay using filter plates is represented as a diagram depicting key assay components and incubation steps. Please click here to view a larger version of this figure.

\section{Biological Sample Preparation}

NOTE: The chosen anatomical site and volume of blood collection is left to the discretion of each individual investigator and will not affect the outcome of the assay. However, once they are obtained, samples should be handled according to the steps listed below.

1. Preparation of Serum Samples

1. Collect the desired volume of blood into preferred collection tube and allow it to clot for at least $30 \mathrm{~min}$.

2. Centrifuge the sample for $10 \mathrm{~min}$ at $1,000 \mathrm{xg}$ at room temperature.

3. Remove serum and assay immediately or aliquot into polypropylene microcentrifuge tubes and store samples at $\leq-20^{\circ} \mathrm{C}$.

\section{Preparation of Plasma Samples}

1. Collect the desired volume of blood using ethylenediaminetetraacetic acid (EDTA) coated blood collection tubes.

2. Centrifuge for $10 \mathrm{~min}$ at $1,000 \times \mathrm{g}$ at room temperature within $30 \mathrm{~min}$ of collection. 
3. Remove plasma and assay immediately, or aliquot into polypropylene microcentrifuge tubes and store samples at $\leq-20^{\circ} \mathrm{C}$.

3. Preparation of Tissue Culture Supernatant Samples

1. Centrifuge the sample for $10 \mathrm{~min}$ at $1,500 \times \mathrm{g}$ at $4{ }^{\circ} \mathrm{C}$ to remove cells and debris.

2. Collect supernatant and assay immediately or aliquot into polypropylene microcentrifuge tubes and store at $\leq-20^{\circ} \mathrm{C}$.

4. Thawing of Frozen Biological Samples

1. Completely thaw any previously frozen biological samples on ice, and vortex briefly before use. Avoid more than 2 freeze/thaw cycles NOTE: The samples used in this protocol were obtained by culturing BALB/c mouse splenocytes $\left(1 \times 10^{6} \mathrm{cells}^{\mathrm{s}} 37^{\circ} \mathrm{C}+5 \% \mathrm{CO}_{2}\right.$ under various conditions: unstimulated, LPS $(100 \mathrm{ng} / \mathrm{mL})$, $\alpha$ CD3 $(1 \mu \mathrm{g} / \mathrm{mL}$ plate-coated $)+\alpha C D 28(1 \mu \mathrm{g} / \mathrm{mL}$ soluble $), P M A(20 \mathrm{ng} / \mathrm{mL})+$ lonomycin $(500 \mathrm{ng} / \mathrm{mL})$. Culture supernatants were collected after $48 \mathrm{~h}$ and processed as described in section 1.3.

\section{Reagent Preparation}

1. Preparation of Pre-mixed Antibody-Immobilized Beads

1. Sonicate pre-mixed beads bottle for $1 \mathrm{~min}$ in a sonicator bath at room temperature, and then vortex for $30 \mathrm{~s}$ prior to use. If no sonicator bath is available, increase the vortex time to $1 \mathrm{~min}$.

2. Preparation of Wash Buffer

1. Bring the 20x Wash Buffer (20x PBS with 1\% Tween-20) supplied with the kit to room temperature and vortex to bring all salts into solution.

2. Dilute $25 \mathrm{~mL}$ of $20 x$ Wash Buffer with $475 \mathrm{~mL}$ deionized water. Store unused portion between $2{ }^{\circ} \mathrm{C}$ and $8{ }^{\circ} \mathrm{C}$ for up to one month.

3. Preparation of Matrix B (For Use with Serum and Plasma Samples Only)

1. Add $5.0 \mathrm{~mL}$ of the Assay Buffer (PBS with $1 \% \mathrm{BSA}$ ) included in the kit to the bottle containing lyophilized Matrix B. Allow at least 15 min for complete reconstitution, then vortex to mix well. Leftover reconstituted Matrix $\mathrm{B}$ can be stored at $\leq-70^{\circ} \mathrm{C}$ for up to one month.

\section{Standard Preparation}

NOTE: Each analyte in this panel has a top standard concentration of $10,000 \mathrm{pg} / \mathrm{mL}$.

1. Add $250 \mu \mathrm{L}$ of Assay Buffer to reconstitute the lyophilized Mouse Th Cytokine Standard Cocktail. Mix by briefly vortexing and allow the vial to sit at room temperature for $10 \mathrm{~min}$.

2. Transfer the standard cocktail to a polypropylene microcentrifuge tube labeled "C7". This will be used as the top standard.

3. Label 6 polypropylene microcentrifuge tubes as C6, C5, C4, C3, C2, and C1.

4. Add $75 \mu \mathrm{L}$ of Assay Buffer to each of these tubes.

5. Transfer $25 \mu \mathrm{L}$ of the top standard $\mathrm{C} 7$ to the $\mathrm{C} 6$ tube and mix well by vortexing. This will be the $\mathrm{C} 6$ standard.

6. Continue performing serial 1:4 dilutions by using a new pipet tip for each tube to add $25 \mu \mathrm{L}$ of the previous standard to the $75 \mu \mathrm{L}$ of Assay Buffer in the next lowest standard tube followed by vortexing to obtain standards C5, C4, C3, C2, and C1. Use Assay Buffer as the 0 pg/mL standard (C0).

\section{Sample Dilution}

NOTE: Preliminary pilot experiments using this assay with multiple dilutions may be required to determine the most appropriate dilution factor for a particular set of biological samples. A proper dilution factor will produce concentration calculations for the sample that lie within the bounds of the standard curve. The following steps are meant as guidelines and may have to be determined empirically depending on sample type.

1. Dilute serum or plasma samples 2-fold with Assay Buffer (e.g. dilute $50 \mu \mathrm{L}$ of sample with $50 \mu \mathrm{L}$ of Assay Buffer) in polypropylene microcentrifuge tubes.

NOTE: If further sample dilution is required, dilutions should be done with Matrix B instead of assay buffer to ensure accurate measurement. Adding serum or plasma samples without dilution will result in low assay accuracy and may clog the filter plate. Matrix B is comprised of pooled mouse serum depleted of endogenous assay targets. It is used as a serum or plasma sample diluent to avoid matrix effects, which are known to the affect analytical sensitivity of immunoassays.

2. Test cell culture supernatant samples without dilution.

NOTE: The levels of analyte can vary greatly from sample to sample. If necessary, dilute supernatant samples using a fresh preparation of their corresponding cell culture medium or Assay Buffer.

\section{Assay Procedure}

NOTE: The assay can be performed in polypropylene filter plates, micro FACS tubes, or V-bottom microplates. The filter plate assay procedure is recommended due to good sample to sample consistency, assay robustness, and ease of handling. This procedure requires a vacuum filtration unit for washing (provided by end-user).

1. Allow all reagents to warm to room temperature $\left(20-25^{\circ} \mathrm{C}\right)$ before use

2. Set the filter plate on an inverted plate cover at all times during the assay setup and incubation steps, so that the bottom of the plate does not touch any surfaces as it may cause leaking.

3. Keep the plate upright during the entire assay procedure, including the washing steps, to avoid losing beads. 
4. Keep the plate in the dark or wrapped with aluminum foil for all incubation steps.

5. Run all standards and samples as duplicates, arranged on the plate in a sequential order.

6. Pre-wet the filter plate by adding $100 \mu \mathrm{L}$ of $1 \mathrm{x}$ Wash Buffer to each well and let it sit for $1 \mathrm{~min}$ at room temperature (if using filter-bottom microplate).

7. Remove buffer volume by using the vacuum manifold (5 - $10 \mathrm{~s}$ ). Do not exceed $10 \mathrm{Hg}$ of vacuum. Blot excess Wash Buffer from the bottom of the plate by pressing the plate on a stack of clean paper towels. Place the plate on top of the inverted plate cover.

NOTE: Steps 5.1 - 5.2 may be omitted if performing the assay using a V-bottom microplate or micro FACS tubes.

8. For cell culture supernatant samples, add $25 \mu \mathrm{L}$ of Assay Buffer to all wells. Add $25 \mu \mathrm{L}$ of each standard to the standard wells. Add $25 \mu \mathrm{L}$ of each sample to the sample wells.

9. For measuring serum or plasma samples, add $25 \mu \mathrm{L}$ of Matrix B to the standard wells. Add $25 \mu \mathrm{L}$ of Assay Buffer to the sample wells. Add 25 $\mu \mathrm{L}$ of each standard to the standard wells. Add $25 \mu \mathrm{L}$ of each diluted serum or plasma sample to the sample wells.

10. Vortex mixed beads for $30 \mathrm{~s}$. Add $25 \mu \mathrm{L}$ of mixed beads to each well, shaking bead bottle intermittently to avoid bead settling. The final volume should be $75 \mu \mathrm{L}$ in each well after the addition of beads.

11. Seal the plate with a plate sealer. Wrap the entire plate, including the inverted plate cover, with aluminum foil. Place the plate on a plate shaker, secure it, and shake at approximately $500 \mathrm{rpm}$ for $2 \mathrm{~h}$ at room temperature. To prevent leaking, do not apply positive pressure to the plate sealer.

12. Without inverting, place the plate on the vacuum manifold and apply vacuum as before and add $200 \mu \mathrm{L}$ of $1 \mathrm{x}$ wash buffer to each well.

13. Remove the contents of the assay plate wells by vacuum filtration. Blot excess wash buffer from the bottom of the plate with an absorbent pad or paper towels. Repeat this step one more time.

NOTE: If the assay is performed using a V-bottom microplate or micro FACS tubes skip steps 5.12 and 5.13 . Instead centrifuge the plate at $1,000 \mathrm{xg}$ for $5 \mathrm{~min}$ at room temperature, then remove the supernatant using a multichannel pipette.

14. Add $25 \mu \mathrm{L}$ of detection antibodies (see the Table of Materials) to each well.

15. Seal the plate with a fresh plate sealer. Wrap the entire plate, including the inverted plate cover, with aluminum foil.

16. Place the plate on a plate shaker and shake at approximately $500 \mathrm{rpm}$ for $1 \mathrm{~h}$ at room temperature.

17. Without vacuuming, add $25 \mu \mathrm{L}$ of SA-PE reagent directly to each well. No dilution of reagent is necessary.

18. Seal the plate with a fresh plate sealer. Wrap the entire plate, including the inverted plate cover, with aluminum foil.

19. Place the plate on a plate shaker and shake at approximately $500 \mathrm{rpm}$ for $30 \mathrm{~min}$ at room temperature.

20. Repeat step 5.13 above.

21. Add $200 \mu \mathrm{L}$ of $1 \times$ Wash Buffer to each well. Re-suspend the beads on a plate shaker for 1 min.

22. Using a multichannel pipet, transfer samples from the filter plate to FACS tubes to read samples on a flow cytometer.

NOTE: Sample volume may be increased from $200 \mu \mathrm{L}$ to $300 \mu \mathrm{L}$ by adding an extra $100 \mu \mathrm{L}$ of $1 \times$ Wash Buffer to each tube to avoid the sample running dry. If necessary samples may be stored at $4{ }^{\circ} \mathrm{C}$ protected from light and analyzed the following day. However, prolonged sample storage can lead to reduced signal.

\section{Flow Cytometer Set-up}

NOTE: In order to generate reliable data, the flow cytometer must be set up properly before data acquisition. This process will vary for individual users in some regards depending on the configuration of the particular instrument the assay is performed on. A generalized setup process for a cytometer able to measure PE and APC and equipped with both a 488 and $633 \mathrm{~nm}$ laser is discussed below.

1. Start up the flow cytometer and acquisition software according to the manufacturer's instructions provided with the instrument.

2. Create a dot plot with FSC (forward scatter) on the X-axis and SSC (side scatter) for the Y-axis. Set FSC and SSC to linear mode.

3. Create a second dot plot with PE on the X-axis and APC for the Y-axis. This plot should be set to a log-based display mode.

4. Vortex the vial of raw beads included in the kit for $30 \mathrm{~s}$ to re-suspend the beads. These beads contain an internal APC dye and are comprised of two size populations.

5. Transfer $400 \mu \mathrm{L}$ of the raw beads to a new FACS tube.

6. Set the flow cytometer flow rate to low.

7. Run the raw beads, carefully adjusting the gain and voltage for FSC and SSC so that both size populations of these beads are visibly separated and easy to gate.

8. Adjust the FSC threshold to exclude unwanted events (i.e. debris or air bubbles).

9. In the FSC vs. SSC plot, draw a gate that includes all bead populations. NOTE: The raw beads contain two size populations of beads, the smaller "A beads" and the larger "B bead" regions.

10. Display the gated bead populations from the FSC vs. SSC plot on the second dot plot with PE on the X-axis and APC for the Y-axis.

11. Adjust the PMT voltage for the APC fluorescence channel so that the APC signal for all bead populations has a median fluorescence intensity (MFI) that lies between $1 \times 10^{1}$ and $5 \times 10^{3}$.

12. Vortex the vial of PE setup beads for $30 \mathrm{~s}$ to re-suspend the beads.

13. Transfer $400 \mu \mathrm{L}$ of the PE beads to a new FACS tube.

14. Replace the raw beads tube from the flow cytometer with the PE beads tube.

15. Adjust the photomultiplier tube (PMT) voltage for the PE fluorescence channel setting so that the MFI of the PE beads falls between the lotspecific range found listed on the PE beads vial. NOTE: The PE setup beads contain beads of one population size ("A beads" only).

\section{Data Acquisition}

NOTE: The exact procedures associated with the acquisition of data on a given instrument can vary and are dependent on the cytometer's configuration specifications and the interface software used. The instructions below are therefore intended to highlight the required steps to be taken in the assay regardless of the cytometer employed in the assay.

1. Verify the cytometer flow rate is still set to low. 
2. Set the number of bead events to be acquired to about 300 per analyte. For a 13 -plex panel this equates to acquiring 3,900 events combined from both bead size populations ( $A+B$ beads).

3. Vortex each sample for $5 \mathrm{~s}$ before analysis.

4. Read samples. When reading samples, set the flow cytometer to setup mode first and wait until bead population is stabilized before switching to acquisition mode.

5. Use simple names with consecutive numbering for data files to facilitate data analysis.

6. Export only the gated events $(A+B$ bead regions) rather than total events.

7. Store all FCS files in the same folder for each assay. If running multiple assays, create a separate folder for each assay.

\section{Proceed to Data Analysis}

NOTE: The FCS files generated on the flow cytometer should be analyzed using the data analysis software, which can be downloaded for free ${ }^{14}$.

1. Install the data analysis software on a PC

2. Transfer all assay FCS files to the computer that contains the analysis software.

3. Plug the license key dongle (included in the kit) into a USB port of the computer.

4. Launch the data analysis software.

5. Click the blue "Add Files" button located at the top of the screen

6. Navigate to the folder that contains the FCS files from the assay in the pop up window that appears.

7. Click and drag all assay FCS files from the pop up window to the software display. All files should now appear in a list.

8. Click the green "Next" button on the bottom right of the display. Left-click and hold to drag the small blue standard curve buttons (C7 to $\mathrm{C} 0$ ) to their corresponding FCS files from the list to define the standard curve.

9. Click the green "Next" button on the bottom right of the display to open the gating pop up window.

10. On the left side of the gating window enter the names of both the A and B bead regions assay analyte targets and their associated bead ID (found in the manual supplied with the assay) in ascending order.

11. Select the gating tool from the top of the pop up window and use it to draw 2 gates in the FSC vs. SSC plot, one around the A beads and a second around the $B$ beads.

12. Review the APC vs. PE scatter plots that appear below the FSC vs. SSC plot that appear. There will be 6 analyte bands in the A beads (left plot) and 7 analyte bands in the $B$ beads (right plot).

NOTE: Gates may be redrawn manually by selecting the eraser tool at the top and clicking to delete a given gate. The gating tool may then be selected and used to manually apply a gate to the desired band region.

13. Click the green "OK" button to close the gating pop up window and return to the list of FCS files.

14. Define any dilutions that were made to biological samples by right clicking a given file, selecting "Dilution Fold" from the menu, and input the correct value.

15. Click the green "Run" button at the bottom right of the display to generate the standard curves for the assay and calculate the concentrations of unknown biological samples.

\section{Representative Results}

This protocol demonstrates how a bead-based immunoassay platform can be used to simultaneously quantitate cytokine profiles from biological samples using a flow cytometer. The biological samples used in this example were cell culture supernatants from mouse splenocytes that had been previously incubated under various activating conditions, although the assay format has also been validated for use with serum or plasma samples.

Data generated from the assay are used to construct standard curves for all analytes in the multiplexed panel. The data analysis software classifies each particular analyte based on a unique bead size and APC intensity, and quantifies them using a PE reporter. The dynamic ranges, as well as the sensitivity of the assay are demonstrated when plotted on a log-log scale in Figure 2A. The software uses assay data and a 5parameter curve fitting algorithm to accurately calculate not only the concentrations of analytes in user provided biological samples but also the limits of detection on both the high and low end of all standard curves (Table 1). The format described in this protocol also provides highly robust assay precision. In Figure 2B and $\mathbf{2 C}$ data are presented depicting the intra-assay variability of each analyte. Two separate standard protein concentrations (high and low) were analyzed in one assay with 16 replicates for each sample. Figure $2 \mathrm{C}$ and $\mathbf{2 D}$ represent the inter-assay variability of target analytes from three independent assays. As with the intra-assay precision experiments, high and low standard concentrations were analyzed with 3 replicates in each of the independent experiments. Minimal variability in the calculated concentration of target proteins is clearly visible.

In order to demonstrate the utility of bead-based cytometric assays in interrogating cytokine expression profiles, mouse splenocytes were incubated under various activating conditions. In addition to an unstimulated control, cells were also incubated with LPS, anti-CD3/anti-CD28 antibodies, or phorbol 12-myristate 13-acetate and ionomycin. Cell culture supernatants were collected $48 \mathrm{~h}$ later and assayed using the Mouse T helper cytokine panel. The quantification results from this experiment are represented in Figure 3 and the effect of stimulation conditions on cytokine concentrations is clearly delineated. 
The results described above are typical, as long as the experimenter has good laboratory technique and follows the provided assay protocol. Sources of error in this assay format can arise from deviations in incubation times, reagent volumes, washing, or from improper PMT settings on the flow cytometer used to analyze the samples. In Figure 4A a scatter plot from a successful assay shows 2 distinct bead populations that are clearly defined. This can be contrasted to Figure 4B, where poor washing and protocol adherence have led to bead aggregates which blur the two populations. In addition, as evidenced by the debris in lower left quadrant, total cytometer events were exported for analysis rather than the preferred format of gated events only. Proper cytometer set up is critical to generating reliable data in this assay. In Figure 4C proper PMT settings allow for the resolution of each of the 6 analytes in the APC classification channel. Improper PMT settings will result in data similar to that presented in Figure 4D where the APC channel has bead populations with overlapping classification intensities. This will invalidate the assay since it will be impossible to calculate analyte concentrations without clearly defined populations of assay beads.

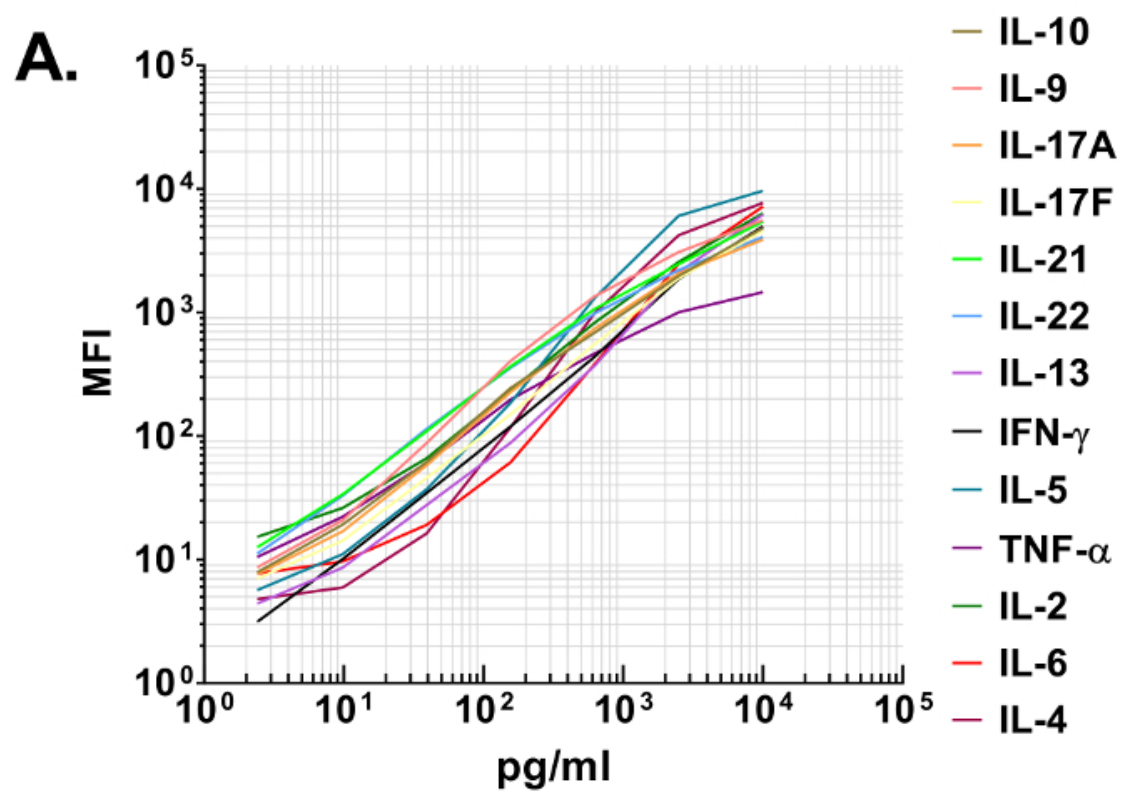

B. C.

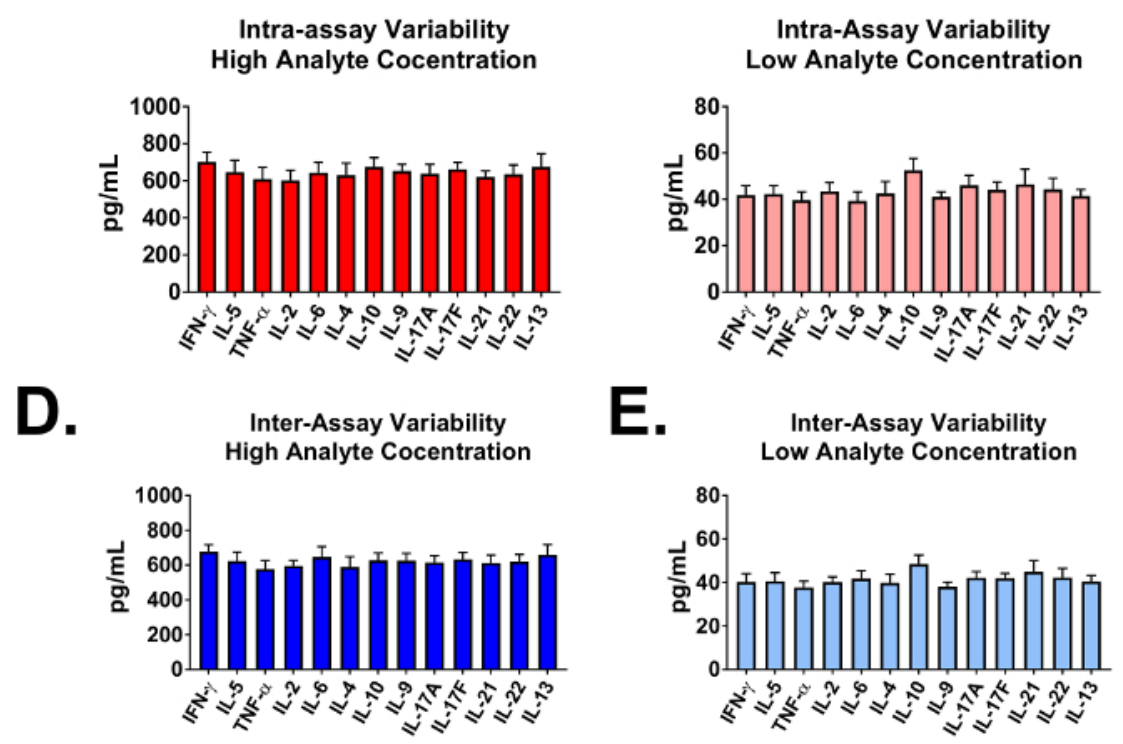

Figure 2: Standard Curve Ranges and Assay Precision. (A) A representative standard curve generated using the Mouse T helper cytokine panel. A standard curve must be run with each assay. (B-C) Intra-assay precision. Two samples with different concentrations of target proteins (high and low) were analyzed in one assay with 16 replicates for each sample. Data are represented as the mean + standard deviation. (D-E) Inter-assay precision. Two samples with different concentrations of target proteins (high and low) were analyzed in three independent assays with 3 replicates for each sample. Data are represented as the mean + standard deviation. Please click here to view a larger version of this figure. 

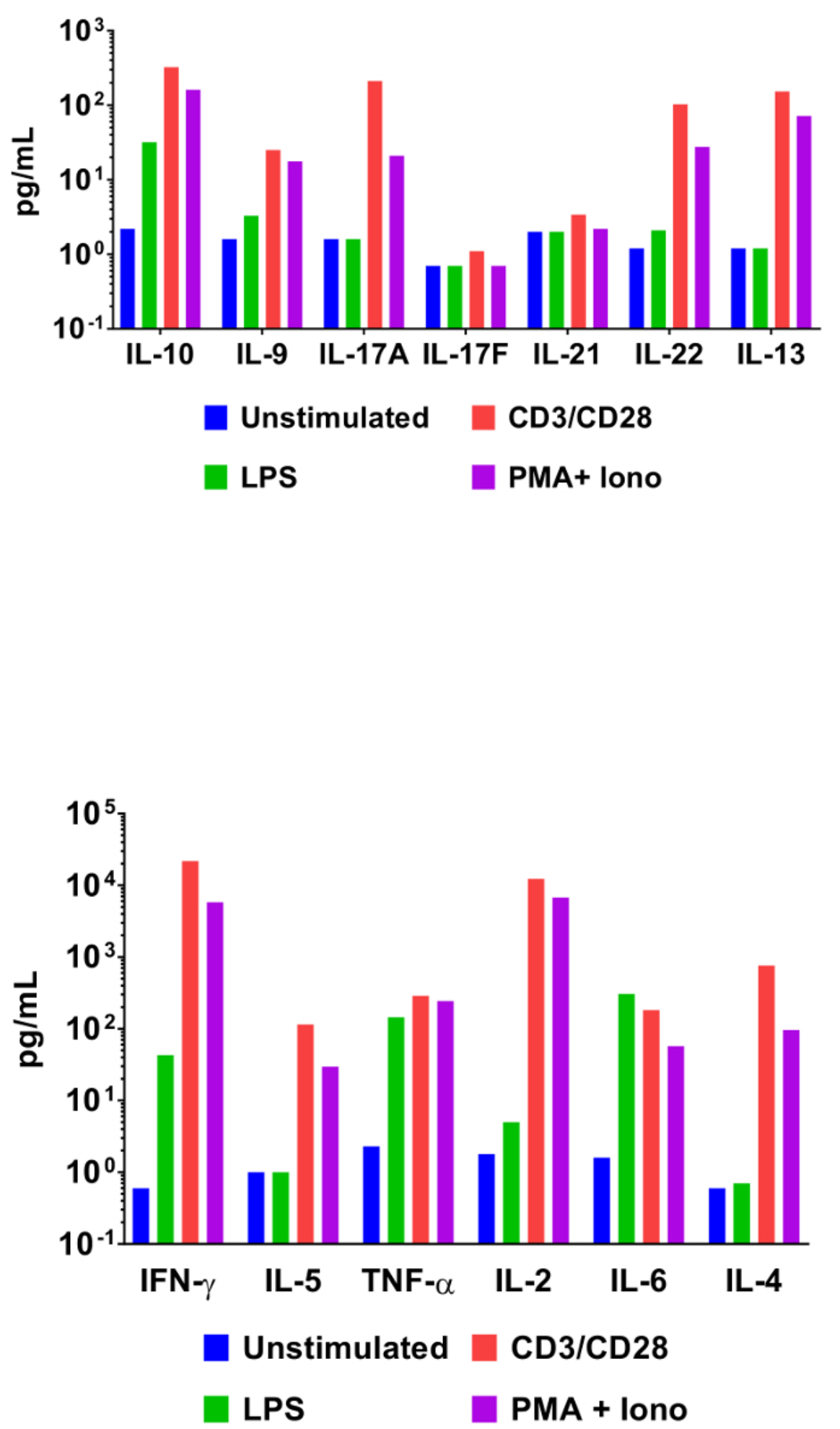

Figure 3: Quantification of Representative Results. Mouse splenocytes $\left(1 \times 10^{6}\right.$ cells) were cultured at $37^{\circ} \mathrm{C}+5 \% \mathrm{CO}_{2}$ under various

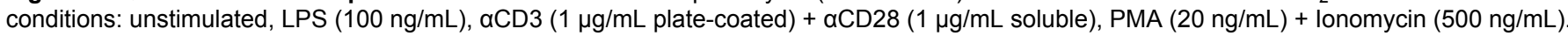
Culture supernatants were collected after $48 \mathrm{~h}$ then quantified using the Mouse T helper cytokine panel. Differential cytokine expression profiles in response to stimulation conditions are clearly visible. Please click here to view a larger version of this figure. 
A.

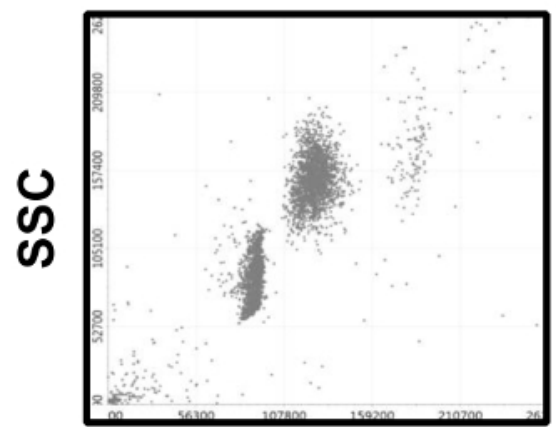

FSC

C.

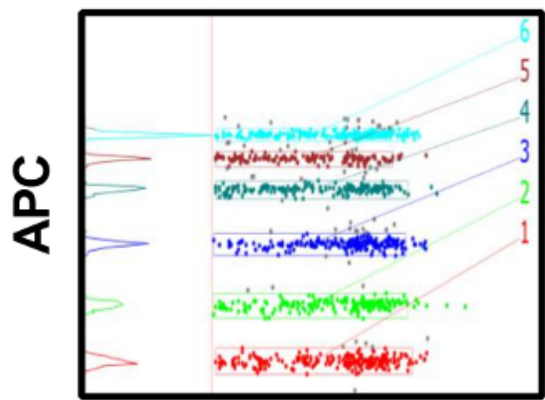

PE
B.

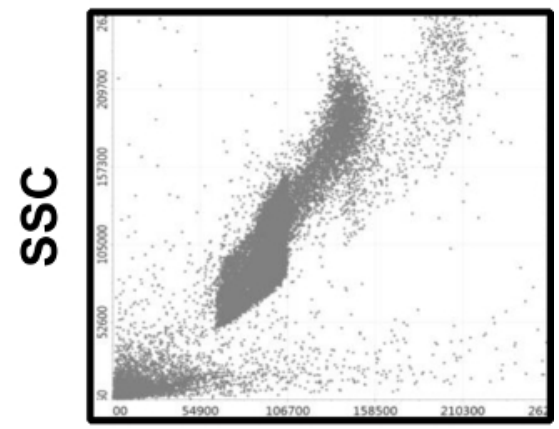

FSC

D.

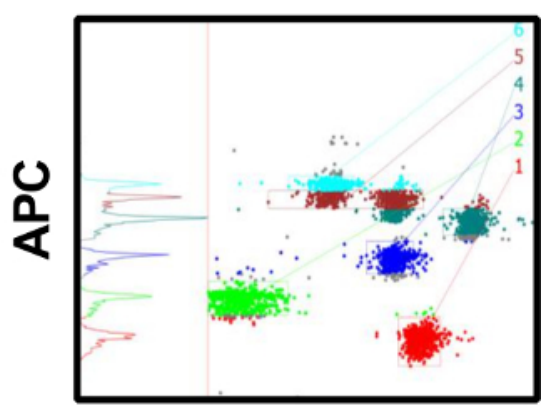

\section{PE}

Figure 4: Results from Successful vs. Unsuccessful Assays. (A) Successful assays will have distinct bead populations for the microsphere populations. (B) Unsuccessful assays will have poor population separation, bead aggregates, and too many collected events. (C) Successful assays have clearly defined intensities in the classification fluorescence channel which are easy to gate and quantify. (D) Unsuccessful assays will have poorly separated and classification intensities which overlap multiple bead populations, making quantification difficult. Many of these errors can be avoided through careful adherence to the assay protocol. Please click here to view a larger version of this figure. 


\begin{tabular}{|c|c|c|c|c|c|}
\hline \multirow[b]{2}{*}{ Analyte } & \multicolumn{3}{|l|}{ Standard Curve } & \multicolumn{2}{|c|}{ Sensitivity (pg/mL) } \\
\hline & Fit Formula & CV & R2 & Min. & Max. \\
\hline IFN-Y & \begin{tabular}{|l|}
$5-P . \log (4.43,14.40$, \\
$0.54,9.11,0.01)$
\end{tabular} & $1.77 \%$ & 0.99 & 2.1 & 18105.0 \\
\hline IL-5 & \begin{tabular}{|l|}
$5-P . \log (4.36,12.68$ \\
$0.64,5.73,0.36)$
\end{tabular} & $1.32 \%$ & 1 & 1.9 & 14514.0 \\
\hline TNF- $\alpha$ & $\begin{array}{l}\text { 5-P.log(4.36, 11.63, } \\
0.79,5.45,0.37)\end{array}$ & $1.48 \%$ & 0.99 & 1.9 & 20109.0 \\
\hline IL-2 & $\begin{array}{l}5-P . \log (4.46,12.62 \\
0.78,5.07,0.34)\end{array}$ & $1.34 \%$ & 1 & 1.9 & 25109.0 \\
\hline IL-6 & \begin{tabular}{|l}
$5-P . \log (4.58,11.66$, \\
$0.64,5.77,0.37)$ \\
\end{tabular} & $1.70 \%$ & 0.99 & 2.0 & 7022.0 \\
\hline IL-4 & \begin{tabular}{|l|}
$5-P . \log (4.27,12.23$, \\
$0.62,5.72,0.36)$ \\
\end{tabular} & $1.36 \%$ & 1 & 2.0 & 13273.0 \\
\hline IL-10 & $\begin{array}{l}\text { 5-P.log(4.67, 11.65, } \\
1.20,6.28,0.61)\end{array}$ & $1.81 \%$ & 0.99 & 2.2 & 5190.0 \\
\hline IL-9 & \begin{tabular}{|l}
$5-P . \log (4.32,13.86$, \\
$0.86,5.90,0.45)$
\end{tabular} & $1.82 \%$ & 1 & 1.9 & 12602.0 \\
\hline IL-17A & $\begin{array}{l}5-P . \log (4.06,14.03 \\
0.47,6.52,0.32)\end{array}$ & $0.99 \%$ & 1 & 2.0 & 12285.0 \\
\hline IL-17F & $\begin{array}{l}5-P . \log (4.52,12.15 \\
0.61,5.52,0.34)\end{array}$ & $0.92 \%$ & 1 & 2.0 & 14841.0 \\
\hline IL-21 & $\begin{array}{l}5-P . \log (4.90,8.03 \\
1.77,4.74,1.11)\end{array}$ & $0.79 \%$ & 1 & 9.0 & 12943.0 \\
\hline IL-22 & \begin{tabular}{|l|}
$5-P . \log (4.57,12.56$, \\
$0.63,6.06,0.39)$
\end{tabular} & $1.16 \%$ & 1 & 2.0 & 6408.0 \\
\hline IL-13 & $\begin{array}{l}5-P . \log (4.40,11.44, \\
0.22,3.19,1.45)\end{array}$ & $1.11 \%$ & 1 & 2.1 & 16728.0 \\
\hline
\end{tabular}

Table 1: Standard Curve Fitting and Assay Sensitivity. The data analysis software was used to generate standard curves for all analytes contained in the multiplex assay. This fully automated analysis applies a robust 5-parameter curve fitting algorithm to the dilution series standards from the assay and was also used to define assay sensitivity using the theoretical limits of detection.

\section{Discussion}

The assay format described in this protocol can provide robust quantification of soluble mediator profiles in biological samples provided the experimenter has good laboratory technique and adheres to the recommended protocol.

There are several key steps that must be followed in order to guarantee reliable results. First, the recombinant standards must be allowed to reconstitute in assay buffer the full time recommended, and after only diluted in polypropylene tubes. Polystyrene tubes can promote the adherence of the proteins to the wall of the tubes, which can lead to low signals when generating the standard curve. Second, proper shaking during incubation steps is critical. Without proper agitation, the binding characteristics of the assay beads can be severely hindered. In addition, proper washing between incubation steps is also required. The experimenter must ensure that excess reagents are removed from the wells prior to commencing the next step in the protocol. Instruments settings for the flow cytometer used to interrogate the assay beads must be optimized as well. In particular, PMT settings must be adjusted to ensure proper bead separation and broad dynamic ranges for the standard curves. Finally, just prior to being analyzed on the cytometer, beads must be vortexed to insure proper suspension and to avoid the formation of aggregates which can skew results.

This protocol can potentially be modified to analyze tissue homogenates as well as the sample types discussed in this manuscript, provided the researcher is willing to optimize their lysis protocol prior to performing large, full plate assays. The actual technique will of course vary depending on tissue type; however, in general the protocol should use a neutral pH buffer containing physiological concentrations of ionic salts, no denaturing chemicals, and preferably no detergents. If detergent must be used, non-ionic detergent concentrations should be kept at a minimum (e.g. no more than 1\%). The buffer should also contain sufficient protease inhibitors to prevent proteolytic degradation of target proteins. Regardless of the lysis protocol used, the final preparations should be centrifuged to remove particulates prior to analysis.

Regarding assay limitations, the concentrations of analyte targets in biological sample types can vary greatly. In order to properly quantify analyte concentrations, they must fall within the top and bottom values for the standard curve. Extrapolation of values of the curve is not necessarily reliable and not recommend. Investigators therefore must optimize dilutions of their particular samples in pilot experiments prior to running a full-plate assay. In addition, the careful preparation of the biological samples to be assayed is paramount to the success of this assay format. Samples that are lipemic, hemolyzed, or contain protein debris will affect the antigen antibody interactions that define the core principle of this immunoassay, and render results which are uninterpretable. 
This assay is significant with respect to existing quantification methods for several reasons. When run properly, the assay format can quantitate up to 13 analytes from a sample using far smaller volumes than would be required to analyze the sample using traditional ELISA assays. This assay format also does not require dedicated instrumentation in order to be performed. This is an advantage compared to other commercially available bead-based immunoassays as the assay can be run using any number of commonly used flow cytometers.

Scientific inquiry into the role played by secreted factors and cytokine networks in health and disease is expanding. The assay format described in this manuscript can be a valuable tool for researchers seeking to understand these multifaceted and complex phenomena. Active biomedical research programs are beginning to explore the role of cytokine networks in not only areas such as generalized inflammation ${ }^{6}$, but also in context of specific disease states such as atherosclerosis, cancer, and neuroinflammation ${ }^{15,16,17}$. Undoubtedly, investigation will continue to grow in these areas as the search for novel therapeutics to treat these chronic conditions expands. The need for accurate and reliable quantification of the soluble mediators associated with these diseases will remain a critical research priority.

\section{Disclosures}

The authors are employed by BioLegend, which manufactures the reagents described in this manuscript. Vigene Tech, Inc. developed the LEGENDplex data analysis software that is used to analyze data generated from the assay described in this manuscript.

\section{Acknowledgements}

The authors would like to acknowledge the contributions of the Biomarkers and Immuno-assays Product Development team to the development of the assay. In addition, we would like to thank Vigene Tech, Inc. for their collaborative efforts in designing the data analysis software packages.

\section{References}

1. Stanley, A. C., \& Lacy, P. Pathways for cytokine secretion. Physiology (Bethesda). 25, 218-229 (2010).

2. Nicola, N. A. Cytokine pleiotropy and redundancy: a view from the receptor. Stem Cells. 12, Suppl 1:3-12; discussion 12-4 (1994).

3. Miyajima, A., Hara, T., \& Kitamura, T. Common subunits of cytokine receptors and the functional redundancy of cytokines. Trends Biochem Sci. 17, 378-382 (1992).

4. Costantini, S., Castello, G., \& Colonna, G. Human Cytokinome: a new challenge for systems biology. Bioinformation. 5, 166-167 (2010).

5. Capone, F. et al. Serum Cytokinome Profile Evaluation: A Tool to Define New Diagnostic and Prognostic Markers of Cancer Using Multiplexed Bead-Based Immunoassays. Mediators Inflamm. 2016, 3064643 (2016).

6. Schett, G., Elewaut, D., McInnes, I. B., Dayer, J. M., \& Neurath, M. F. How cytokine networks fuel inflammation: Toward a cytokine-based disease taxonomy. Nat Med. 19, 822-824 (2013).

7. Tse, E., \& Kwong, Y. L. T-cell lymphoma: Microenvironment-related biomarkers. Semin Cancer Biol. 34, 46-51 (2015).

8. Reale, M., Greig, N. H., \& Kamal, M. A. Peripheral chemo-cytokine profiles in Alzheimer's and Parkinson's diseases. Mini Rev Med Chem. 9, 1229-1241 (2009).

9. Vistnes, M., Christensen, G., \& Omland, T. Multiple cytokine biomarkers in heart failure. Expert Rev Mol Diagn. 10, 147-157 (2010).

10. Gobel, K. et al. Blood coagulation factor XII drives adaptive immunity during neuroinflammation via CD87-mediated modulation of dendritic cells. Nat Commun. 7, 11626 (2016).

11. Palm, A. K., Friedrich, H. C., \& Kleinau, S. Nodal marginal zone B cells in mice: a novel subset with dormant self-reactivity. Sci Rep. 6, 27687 (2016).

12. Yu, Y. et al. The transcription factor Bcl11b is specifically expressed in group 2 innate lymphoid cells and is essential for their development. $J$ Exp Med. 212, 865-874 (2015).

13. Pelly, V. S. et al. IL-4-producing ILC2s are required for the differentiation of TH2 cells following Heligmosomoides polygyrus infection. Mucosal Immunol. 9, 1407-1417 (2016).

14. LEGENDplex Multiplex Assays. <https://www.biolegend.com/legendplex> (2017).

15. Autieri, M. Pro- and anti-inflammatory networks in atherosclerosis. ISRN Vascular Medicine. 2012, Article ID 987629, 17 pages (2012).

16. West, N. R. et al. Emerging cytokine networks in colorectal cancer. Nat Rev Immunol. 15, 615-629 (2015).

17. Becher, B., Spath, S., \& Goverman, J. Cytokine networks in neuroinflammation. Nat Rev Immunol. 17, 49-59 (2017). 